\title{
Evaluating Ketosis in Primate Field Studies: Validation of Urine Test Strips in Wild Bornean Orangutans (Pongo pygmaeus wurmbii)
}

\author{
Daniel J. Naumenko ${ }^{a-c}$ Malcolm Watford $^{d}$ Sri Suci Utami Atmoko ${ }^{\mathrm{e}}$ \\ Wendy M. Erbb, c Erin R. Vogel ${ }^{\text {b, c }}$ \\ a Department of Anthropology, University of Colorado, Boulder, CO, USA; ${ }^{b}$ Department of \\ Anthropology, Rutgers University, New Brunswick, NJ, USA; ${ }^{\circ}$ Center for Human Evolutionary \\ Studies, Rutgers University, New Brunswick, NJ, USA; d Department of Nutritional Sciences, \\ Rutgers University, New Brunswick, NJ, USA; ${ }^{~}$ Fakultas Biologi, Universitas Nasional, Jakarta, \\ Indonesia
}

\author{
Keywords \\ Ketones · Energetics · Urinalysis · Chemstrips ${ }^{\circledR}$
}

\begin{abstract}
The use of urine test strips (e.g., Roche Chemstrip ${ }^{\circledR}$ ) has become the standard for quickly assessing the physiological condition and/or health of wild primates. These strips have been used to detect ketosis as a marker of fat catabolism in several primate taxa in their natural environments in response to changing food availability. However, the use of urine strips to determine ketosis has only been validated in human studies, and thus it remains unclear whether these strips accurately detect and quantify ketone bodies in nonhuman primates. We examined variations in ketone body concentrations in urine samples collected from wild Bornean orangutans at the Tuanan Orangutan Research Station. We assessed the accuracy of qualitative results from Chemstrip test strips in the field (i.e., negative, small, moderate, and large) using an enzyme-linked assay in the laboratory to determine the concentrations of acetoacetate of the same urine samples. Urine samples that tested positive for ketones in the field had significantly higher levels of ketones in the enzymatic assay compared to those that tested negative. There was significant variation in acetoacetate concentrations among the 4 Chemstrip values; however, post hoc tests revealed no significant differences between negative and small samples. We conclude that urinary test strips provide a useful tool for determining ketotic state in wild orangutans, but caution should be taken when interpreting results from samples showing only small levels of ketones on these strips.

(c) 2019 S. Karger AG, Basel
\end{abstract}

KARGER

(c) 2019 S. Karger AG, Basel

karger@karger.com www.karger.com/fpr
Daniel J. Naumenko

Department of Anthropology, University of Colorado 1350 Pleasant St. Hale 350, UCB 233

Boulder, CO 80309-0233 (USA)

E-Mail daniel.naumenko@ colorado.edu

Dr. Erin R. Vogel

Department of Anthropology, Rutgers University Ruth Adams Building, 3rd Floor, 131 George St. New Brunswick, NJ 08901 (USA) E-Mail erin.vogel@rutgers.edu 


\section{Introduction}

Variation in food availability has pronounced effects on primate feeding behavior and physiology in the wild [Knott, 1998; Lambert and Rothman, 2015; Vogel et al., 2015, 2017a; Emery Thompson, 2017]. This variation, when extreme or unpredictable, may result in a negative energy balance in which energy expenditure exceeds intake [Knott, 1998]. A prolonged negative energy balance can have long-term fitness consequences if an individual does not have energy reserves to meet their daily energetic requirements [Hoffman et al., 2008; Archie et al., 2014]. At times of reduced food intake, fatty acids derived from adipose triglyceride stores can be partially oxidized in the liver to produce ketone bodies as an alternative fuel for tissues such as the brain. In the absorptive state, circulating ketone body concentrations are usually less than $0.5 \mathrm{mM}$ in humans, but when fatty acids become the dominant metabolic fuel, circulating ketone levels steadily rise [Laffel, 1999]. Plasma ketone concentrations greater than $1 \mathrm{~mm}$ are often used to define physiological ketosis, although prolonged starvation and uncontrolled type 1 diabetes can result in much higher ketone body concentrations [Robinson and Williamson, 1980; Laffel, 1999]. As the concentration of ketone bodies rises in the plasma, excretion from plasma into the urine allows for a noninvasive and readily available sample for quantitative analysis. Urinary ketone levels can therefore indicate ketosis, a state characterized by elevated ketone body levels [Laffel, 1999], caused by a negative energy balance, which can be linked to food scarcity in the wild.

When fat is catabolized for energy during ketogenesis, the process by which fatty acids are converted to ketone bodies [Laffel, 1999], 3 different ketone bodies are produced: acetone, $\beta$-hydroxybutyrate, and acetoacetate. Due to its volatility and lower abundance compared to the other 2 ketone bodies [Laffel, 1999], acetone is difficult to measure and consequently it is often excluded from analyses. The latter $2 \mathrm{can}$ be readily measured in vitro using $D$ - $\beta$-hydroxybutyrate-dehydrogenase which interconverts the 2 . D- $\beta$-Hydroxybutyrate-dehydrogenase reduces acetoacetate with the help of NADH, producing $\beta$-hydroxybutyrate and NAD+. This method has been described in greater detail by Williamson et al. [1962].

Chemstrips ${ }^{\circledR}$ are a common method for semi-quantitatively measuring ketones in urine collected noninvasively from nonhuman primates [Knott, 1998; Sleeman and Mudakikwa, 1998; Kaur and Huffman, 2004; Krief et al., 2005; Emery Thompson and Knott, 2008; Beaman et al., 2009; Harrison et al., 2010; Leendertz et al., 2010; MacIntosh et al., 2012]. They provide a quick and cost-efficient way to measure acetone and acetoacetate and thus assess negative energy balances in the field, though acetone will generally evaporate rapidly due to its volatility. However, these test strips were designed for use in clinical settings on humans and have not been validated for use in the field on nonhuman primates. Our objective was to validate these strips using orangutans as a model species, as they have been the subjects of long-term studies in the wild that have used urinary test strips to measure ketones [Knott, 1998; Emery Thompson and Knott, 2008; Harrison et al., 2010; Vogel et al., 2015; Naumenko et al., 2016; Erb et al., 2018].

Bornean orangutans are an excellent model for validating ketone measurement by urinary test strips as their Southeast Asian forest habitats are characterized by unpredictable and extreme fluctuations in the availability of fruit, orangutans' preferred source of calories [Vogel et al., 2008]. Bornean orangutans have been shown to expe- 
rience a $75 \%$ reduction in caloric intake during episodes of fruit scarcity [Vogel et al., 2017a], relying on low-calorie fallback foods [Harrison et al., 2010] and energy from fat stores to survive. Knott [1998] found that wild orangutans use fat stores in response to low fruit availability, and Emery Thompson and Knott [2008] showed that the production of urinary ketone bodies and C-peptide of insulin, a biomarker of energy balance, are negatively correlated, indicating the use of fat stores to supplement energetic needs when the dietary intake is reduced. Although Chemstrips have been used to document the presence of ketones in the urine of orangutans and other nonhuman primate species [e.g., macaques: MacIntosh et al., 2012; gibbons: Beaman et al., 2009; gorillas: Sleeman and Mudakikwa, 1998; and chimpanzees: Kaur and Huffman, 2004; Krief et al., 2005; Leendertz et al., 2010], the quantitative range of concentrations represented by each qualitative ketone category remains unknown. Here, we present the first validation in a nonhuman primate species of this Chemstrip field method, doing so in a wild Bornean orangutan population. We compared field results using urinary Chemstrips to laboratory results using an in vitro enzyme-linked assay to examine whether: (1) the presence or absence of ketones using urinary Chemstrips accurately reflects the concentrations of acetoacetate and (2) the Chemstrip categories for ketones can be distinguished from each other.

\section{Methods}

Study Area and Sample Collection

Urine samples were collected from 2011 to 2014 from wild orangutans at the Tuanan Orangutan Research Station ( $2^{\circ} 09^{\prime} \mathrm{S}$ and $\left.114^{\circ} 26^{\prime} \mathrm{E}\right)$ in the 3,090 $\mathrm{km}^{2}$ Mawas Conservation Area in Central Kalimantan, Indonesia. These samples are part of an ongoing, longitudinal study focusing on orangutan energetics and health. Urine was collected by stretching transparent plastic over a forked stick and catching the first morning void as soon as the orangutan left its morning nest [Knott, 1997]. Urine was pipetted into $15-\mathrm{mL}$ centrifuge tubes and samples were stored on ice in a thermos until they could be brought back to camp, where they were aliquoted and stored at $-20^{\circ} \mathrm{C}$ for shipment. Frozen samples were exported to the Laboratory for Primate Dietary Ecology and Physiology at Rutgers University (USA), where they were stored at $-80{ }^{\circ} \mathrm{C}$ until analyzed in the spring of 2016.

For this study, we utilized urine samples $(n=136)$ from 30 known individuals ( 20 males and 10 females; mean $[ \pm \mathrm{SD}]$ urine samples per individual: $4.39 \pm 4.36$, range: $1-18)$. Samples were included in the analysis only if they: were first morning voids, were stored on ice prior to freezing at the field station, were not noted as appearing to contain blood, and did not undergo a freezethaw cycle in the laboratory for use in another assay. Since samples testing negative for ketones were more abundant, we a priori selected a target number roughly equal to the number of positive samples (72 negative and 64 positive). We additionally aimed to include negative samples across periods varying in the fruit availability index (FAI; the percent of trees producing fruits each month in phenology plots at Tuanan; $n=17$ (low), 47 (medium), and 8 (high), as this index provides a reasonable proxy for caloric intake in wild Bornean orangutans, except flanged males [Vogel et al., 2017a].

\section{Ketone Measurements}

At the time of sample collection in the forest, a small volume of urine was pipetted directly onto Chemstrip (Roche Chempstrip ${ }^{\mathrm{TM}}$ 10UA) test pads. After $60 \mathrm{~s}$, observers compared the change in color in the ketone test area against the corresponding color chart on the Chemstrip container. Results were recorded according to the manufacturer's instructions as: negative (beige, no color change), small (light violet), moderate (medium violet), and large (dark violet). All field assistants were trained in interpretation of Chemstrip results by Dr. Erin Vogel in the field, and,

Validation of Ketone Urine Test Strips in Wild Bornean Orangutans
Folia Primatol 2020;91:159-167 DOI: 10.1159/000501933 
if there was any doubt regarding the result, the 2 members of the field team jointly decided on the color. To prevent issues resulting from high environmental humidity, Chemstrips were stored in capped bottles with a small packet of silica gel in the field and in airtight containers with silica gel when not in use. Silica gel was checked regularly and changed when the silica changed color, indicating saturation. Since highly pigmented urine can lead to false-positive readings [Comstock and Garber, 1990], we conducted 2 sets of tests: 1 that included and 1 that excluded darkly pigmented samples $(n=8)$. When the darkly pigmented samples were omitted from the analyses, the differences between the negative and positive samples were still significant.

Acetoacetate and $\beta$-hydroxybutyrate concentrations were quantified by enzymatic ( $\beta$-hydroxybutyrate dehydrogenase; Sigma $\left.{ }^{\circledR}\right)$ reduction of NAD $+(\beta$-hydroxybutyrate) or oxidation of NADH (acetoacetate) according to the methods of Williamson et al. [1962], briefly described below, at the Department of Nutritional Sciences, Rutgers University. Results were expressed in millimolar (mM). For the estimation of acetoacetate, we added the following mixture to a $1-\mathrm{cm}$ cuvette: potassium phosphate buffer $(100 \mu \mathrm{mol} ; 1 \mathrm{~mL}$ of $0.1 \mathrm{M}$; pH 7.0), NADH $(0.5 \mu \mathrm{mol} ; 0.1 \mathrm{~mL}$ of $5 \mathrm{mM}$ ), undiluted urine sample $(50 \mu \mathrm{L})$, and distilled water to a final volume of $3 \mathrm{~mL}$. Cuvettes were covered with parafilm and inverted, and then read at $340 \mathrm{~nm}$ in a spectrophotometer every 5-10 min until the reading was stable. $\beta$-Hydroxybutyrate dehydrogenase $\left(25 \mu \mathrm{L}\right.$; Sigma $\left.{ }^{\circledR}\right)$ was then added to each cuvette, inverted, and incubated at room temperature for $30 \mathrm{~min}$, after which they were read every $10 \mathrm{~min}$ until stable. The estimation of $\beta$-hydroxybutyrate is analogous to that of acetoacetate, with a few minor modifications, as follows. The following mixture was added to a 1 -cm cuvette: Tris buffer $(50 \mu$ mole; $0.5 \mathrm{~mL}$ of $0.1 \mathrm{M}$; pH 8.5$), \mathrm{NAD}+(1 \mu \mathrm{mol} ; 0.1 \mathrm{~mL}$ of $10 \mathrm{mM}$ ), hydrazine hydrate ( $1 \mathrm{~mL}$ of the following mixture: $1 \mathrm{~mL}$ hydrazine hydrate, $5 \mathrm{~mL} \mathrm{HCl}$, diluted to $20 \mathrm{~mL}$ with distilled water), urine sample ( $50 \mu \mathrm{L})$, and distilled water to a final volume of $3 \mathrm{~mL}$. All other steps were identical to acetoacetate estimation, though the readings took longer to stabilize. Distilled water of equal volume was used as a blank in both assays. The limit of detection for these assays is $0.01 \mathrm{mM}$, which is effectively zero.

\section{Statistical Analyses}

Acetoacetate concentrations were log-transformed (base 10) to achieve normality (ShapiroWilk, $p=0.44$ ), and ANOVA was used to test for differences in mean acetoacetate concentrations among the 4 ketone test strip categories. Two samples were excluded from the statistical analysis: one due to experimental error leading to a value of 0 for both acetoacetate and $\beta$-hydroxybutyrate, and the second due to insufficient volume to measure the acetoacetate concentration. Welch's $t$ test was used to test for a difference between negative and all positive samples. Spearman's $\rho$ was used to assess the correlation between untransformed acetoacetate and $\beta$-hydroxybutyrate. Although Chemstrips do not measure $\beta$-hydroxybutyrate, it was possible that $\beta$-hydroxybutyrate might be present at high levels, even if acetoacetate was low. In such a case, the Chemstrip might give a false negative. It was thus necessary to check that both ketone bodies tracked with each other to ensure against such false negatives. To correct for the 6 post hoc multiple comparisons, a Holm-Bonferroni adjustment was used [Holm, 1979]. We set the significance level for all tests to $\alpha<0.05$. All statistical procedures were conducted in R 3.5.1 (The R Foundation for Statistical Computing: http://www.R-project.org).

\section{Results}

$\beta$-Hydroxybutyrate values were positively correlated with acetoacetate values in the assayed samples (Spearman's $\rho=0.46, p<0.0001$ ). All subsequent analyses were thus performed on acetoacetate values only, since Chemstrips do not measure $\beta$-hydroxybutyrate. The mean $( \pm \mathrm{SE})$ acetoacetate concentration assayed enzymatically ranged from $0.297 \pm 0.037 \mathrm{mM}$ for samples testing as negative to $2.212 \pm$ $0.378 \mathrm{mM}$ for samples testing as large using Chemstrips (Table 1). Samples testing negative differed significantly from those testing positive (small, moderate, and large 
Fig. 1. Boxplot depicting enzyme-linked assay results of acetoacetate concentrations across 4 Chemstrip test categories for ketones.

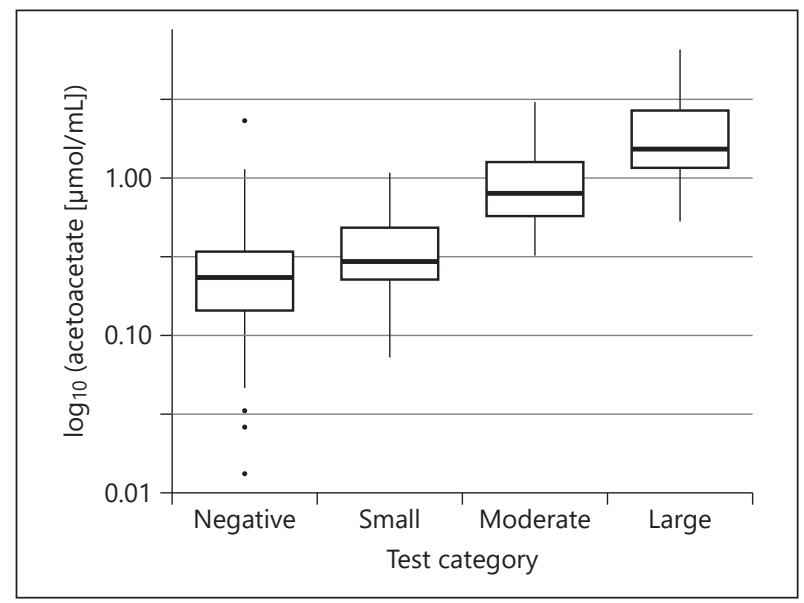

Table 1. Acetoacetate concentrations for each urinary test strip category

\begin{tabular}{llll}
\hline Test strip result & Mean & SE of the mean & 95\% CI \\
\hline Negative & 0.297 & 0.037 & $0.223-0.370$ \\
Small & 0.376 & 0.049 & $0.278-0.474$ \\
Negative-small $^{1}$ & 0.317 & 0.03 & $0.256-0.377$ \\
Moderate & 0.984 & 0.141 & $0.701-1.266$ \\
Large & 2.212 & 0.378 & $1.456-2.969$ \\
\hline
\end{tabular}

Means were taken from untransformed values of acetoacetate concentration. ${ }^{1}$ This category was recalculated after samples were merged due to the lack of significant differences between the 2 categories.

combined; Fig. 1; Welch's $t$ test, $t=-7.837, p<0.0001)$. Furthermore, there was significant variation across all 4 qualitative Chemstrip ketone categories (Fig. 1; ANOVA, $\left.F_{3,132}=46.36, p<0.0001\right)$. Post hoc multiple comparisons revealed that there were significant differences among all qualitative values except between negative and small (Fig. 1; Tukey's HSD with Holm-Bonferroni adjustment, negative-small: $p=$ 0.126 ; all other comparisons: $p<0.03$ ).

\section{Discussion}

Test strip measurements of urinary ketones provide valuable information about fat catabolism in wild orangutans. However, this study raises important methodological considerations for future nonhuman primate energetic studies. First, the negative category is somewhat misleading in that it does not necessarily imply a total absence of ketone bodies in urine. All assayed samples had acetoace- 
tate present at a detectable level, indicating that the presence of at least low levels of ketones in negative samples cannot be discounted, and their presence may even be ubiquitous, which fits with expectations [Laffel, 1999; Roche Diagnostics, 2013]. Second, since the negative and small categories of ketones could not be statistically differentiated on the basis of their acetoacetate concentrations, they should be considered as a single group distinct from both the moderate and large categories (Table 1) in future orangutan studies. The binned negative-small category indeed encompasses a range of ketone body values below the $0.5 \mathrm{~mm}$ normal level of circulating ketone body concentrations, while the moderate and large samples exceed this threshold. Third, the large category represents an extensive range of ketone body concentrations with an as yet unidentified upper bound. Though our highest result for acetoacetate concentration from the enzymatic assay was $6.43 \mathrm{mM}(13.49 \mathrm{mM}$ with $\beta$-hydroxybutyrate combined), we cannot conclude that this is the maximum possible value, as concentrations could potentially reach higher levels depending on the severity of the dietary energy deficit and the species in question. Finally, Chemstrips appear to provide a reasonable measure of total ketone bodies (acetoacetate and $\beta$-hydroxybutyrate combined) given that the two ketone bodies were positively correlated with each other.

There are a few limitations of this study. We note that there was a delay between field assessment of ketone levels and laboratory analysis, which may be a source of error. This time difference may account for the lack of distinction between negative and small categories if the ketone body stability was compromised in the interim. Additionally, field results were collected by a variety of field assistants, volunteers, and researchers, and interpretation of Chemstrip color changes may have been an additional source of error, despite training and using the same color key. We encourage good interobserver reliability field practices in on-going and future nonhuman primate studies. Although the quantitative means and ranges are likely applicable to other orangutan populations, the specific values we documented here may not apply directly to other nonhuman primates. Furthermore, the ranges presented here may indeed be larger for orangutans, as indicated by the unidentified upper bound of the large category. It is possible that longer or more severe fruit shortages, habitats of a lower nutritional quality, or other unknown factors might lead to even higher ketone levels in urine than this study detected. We must also note the interpretive caveat that although ketones are expected to increase in relation to negative energy balance they are not diagnostic of it. Ketones increase in situations of glucose deficiency, so the relationship between negative energy balance and ketones also depends on both carbohydrate intake and fat availability, either via diet or via body stores [Laffel, 1999].

Several studies have used urine Chemstrips to assess ketone levels in nonhuman primates. In the single study of a nonape species, fewer than one third of macaque urine samples tested positive for ketones, with the majority of those (96\%) detecting only small values [MacIntosh et al., 2012]. Similarly, at Kibale and Mahale, chimpanzee urine samples testing positive for ketones were extremely rare [Kaur and Huffman, 2004; Krief et al., 2005], whereas chimpanzees in West Africa appeared to have a slightly greater prevalence [Leendertz et al., 2010]. The prevalence of samples testing negative in these studies, with much of the remainder testing as small, suggests that these populations do not suffer prolonged periods of dietary energy deficiency. Further, given the methodological considerations revealed in the present study, caution must be taken when interpreting "positive" binned results, particularly samples 
with small ketone levels. Pooling small ketones with medium and large categories as "positive" is not advised.

Previous studies of 3 wild Bornean orangutan populations have detected ketones in urine using the same Chemstrips validated in this study [Knott, 1998; Harrison et al., 2010; Vogel et al., 2017b]; the results of this validation therefore apply to each of these studies. Given that these 3 studies reported conflicting findings on the relationship between fruit availability, energy intake, and ketone levels, it is possible that different populations of the same species have different strategies for maintaining energy balance or that differences in habitat drive the use of fat catabolism as a back-up energy source. At Gunung Palung, Knott [1998] documented the expected negative relationship between fruit availability (FAI) and ketones, detecting positive samples only during low FAI periods and only negative samples during masting months when the FAI was high. Long-term research at Tuanan has similarly documented a negative relationship between FAI and ketones [Vogel et al., 2017b]. Harrison et al. [2010], however, found no correlation between ketones and FAI for adult females and flanged males at Sabangau despite a range of FAI values similar to those observed at Gunung Palung [Knott, 1998] and Tuanan [Vogel et al., 2017b]. Harrison et al. [2010] suggested these results might be due to the lack of masting at their site; their average monthly ketone scores support this statement, as they are almost all negative and small and thus part of the same group. Two of these studies [Knott, 1998; Harrison et al., 2010] did not indicate whether they restricted their analyses to first morning void urine samples, and it is possible that collection of urine samples at different times of day may have contributed to the discrepancy in findings. As such, it would be interesting to reassess these data, accounting for the results of this validation, to better understand the relationship between environmental fruit availability and ketone production in these populations. Our results highlight the need to integrate additional methods of assessing energy intake and energy balance (e.g., caloric intake and urinary C-peptide of insulin) to complement urine strip ketone measures and deepen our understanding about the relationship between environmental variation, energy balance, and ketogenesis.

As the physiology and biochemistry of ketogenesis should be highly conserved across mammals [Williamson and Whitelaw, 1978; Robinson and Williamson, 1980], we expect that the validation of this field method should be applicable to other nonhuman primate species. We encourage other primate researchers to follow the protocol described in this study for energetic studies, and efforts should be made to utilize urine samples that represent biologically relevant ranges of caloric intake and energetic balance. Although test strips should accurately detect urinary ketones across nonhuman primates, species-specific validations are recommended to determine the precise ranges of ketone body concentration that correspond to each test strip category.

We conclude that Chemstrips offer a useful noninvasive method for monitoring wild orangutan fat catabolism and ketone levels in the context of other nutritional and energetic data. Caution must be exercised when interpreting differences in qualitative ketone level categories, as some may overlap with each other, or represent large ranges. It is therefore important that studies of ketone levels in species other than humans or orangutans include a validation of the method. Further research on wild orangutans will explore how ketone bodies vary with food availability and abundance and incorporate them into broader studies of energetics.

Validation of Ketone Urine Test Strips in Wild Bornean Orangutans
Folia Primatol 2020;91:159-167 DOI: $10.1159 / 000501933$ 


\section{Acknowledgement}

We thank the Tuanan team of assistants (Abuk, Idun, Isman, Rahmadt, Suwi, and Tono), staff, and all participating students for their efforts in urine sample and data collection. We are grateful to Tatang Mitra Setia, Astri Zulfa, and Maria van Noordwijk for their collaborative research at Tuanan. We gratefully acknowledge the Indonesian State Ministry for Research, Technology and Higher Education (RISTEK-DIKTI), the Indonesian Institute of Sciences (LIPI), the Directorate General of Forest Protection and Nature Conservation (PHKA), the Ministry of Internal Affairs, the Nature Conservation Agency Palangkaraya (BKSDA), the local government in Central Kalimantan, and the Borneo Orangutan Survival Foundation (BOSF Indonesia and BOSF Mawas) for their permission and support of this project.

\section{Statement of Ethics}

The observation and collection protocols used were approved by the IACUC committee of Rutgers (protocol No. 11-030).

\section{Disclosure Statement}

The authors have no conflict of interests to declare.

\section{Funding Sources}

This research was funded by USAID (APS-497-11-000001 to E.R.V.) for sample collection and transport, field site upkeep, urine test strips, and other related expenses, and by the NJ Agricultural Experimental Station (project No. 14175 to M.W.) for laboratory analyses.

\section{Author Contributions}

Daniel J. Naumenko performed all laboratory analyses, analyzed data, and wrote this paper. Malcolm Watford provided training and expertise in laboratory analyses and background, equipment and reagents for said analyses, and revisions/edits of this paper. Sri Suci Utami Atmoko provided critical assistance in acquisition of permits, storage of samples during transport, and project administration. Wendy $\mathrm{M}$. Erb helped to conceptualize the project, collected and exported samples, and revised/edited this paper. Erin R. Vogel established and maintained field collections including funding and project supervision, conceptualized the project, and revised/edited this paper.

\section{References}

Archie EA, Altmann J, Alberts SC (2014). Costs of reproduction in a long-lived female primate: injury risk and wound healing. Behavioral Ecology and Sociobiology 68: 1183-1193.

Beaman BA, Hesemeyer WJ, Dominy NJ, Savini T, Reichard UH (2009). Sterile pyuria in a population of wild white-handed gibbons (Hylobates lar). American Journal of Primatology 71: 880-883.

Comstock JP, Garber AJ (1990). Ketonuria. In Clinical Methods: the History, Physical, and Laboratory Examinations (Walker HK, Hall WC, Hurst JW, eds.), pp 658-661. Boston, Butterworths.

Emery Thompson M (2017). Energetics of feeding, social behavior, and life history in non-human primates. Hormones and Behavior 91: 84-96. 
Emery Thompson M, Knott CD (2008). Urinary C-peptide of insulin as a non-invasive marker of energy balance in wild orangutans. Hormones and Behavior 53: 526-535.

Erb WM, Barrow EJ, Hofner AN, Utami-Atmoko SS, Vogel ER (2018). Wildfire smoke impacts activity and energetics of wild Bornean orangutans. Scientific Reports 8: 7606.

Harrison ME, Morrogh-Bernard HC, Chivers DJ (2010). Orangutan energetics and the influence of fruit availability in the nonmasting peat-swamp forest of Sabangau, Indonesian Borneo. International Journal of Primatology 31: 585-607.

Hoffman CL, Ruiz-Lambides AV, Davila E, Maldonado E, Gerald MS, Maestripieri D (2008). Sex differences in survival costs of reproduction in a promiscuous primate. Behavioral Ecology and Sociobiology 62: 1711.

Holm S (1979). A simple sequentially rejective multiple test procedure. Scandinavian Journal of Statistics 6: 65-70.

Kaur T, Huffman MA (2004). Descriptive urological record of chimpanzees (Pan troglodytes) in the wild and limitations associated with using multi-reagent dipstick test strips. Journal of Medical Primatology 33: 187-196.

Knott CD (1997). Field collection and preservation of urine in orangutans and chimpanzees. Tropical Biodiversity 4: 95-102.

Knott CD (1998). Changes in orangutan caloric intake, energy balance, and ketones in response to fluctuating fruit availability. International Journal of Primatology 19: 1061-1079.

Krief S, Huffman MA, Sévenet T, Guillot J, Bories C, Hladik CM, Wrangham RW (2005). Noninvasive monitoring of the health of Pan troglodytes schweinfurthii in the Kibale National Park, Uganda. International Journal of Primatology 26: 467-490.

Laffel L (1999). Ketone bodies: a review of physiology, pathophysiology and application of monitoring to diabetes. Diabetes/Metabolism Research and Reviews 15: 412-426.

Lambert JE, Rothman JM (2015). Fallback foods, optimal diets, and nutritional targets: primate responses to varying food availability and quality. Annual Review of Anthropology 44: 493-512.

Leendertz SAJ, Metzger S, Skjerve E, Deschner T, Boesch C, Riedel J, Leendertz FH (2010). A longitudinal study of urinary dipstick parameters in wild chimpanzees (Pan troglodytes verus) in Cote d'Ivoire. American Journal of Primatology 72: 689-698.

MacIntosh AJ, Huffman MA, Nishiwaki K, Miyabe-Nishiwaki T (2012). Urological screening of a wild group of Japanese macaques (Macaca fuscata yakui): investigating trends in nutrition and health. International Journal of Primatology 33: 460-478.

Naumenko DJ, Watford M, Erb WM, Vogel ER (2016). Validation of urine test strips to measure relative ketone body levels in wild Bornean orangutans, Pongo pygmaeus wurmbii. American Journal of Physical Anthropology 159: 238-239.

Robinson AM, Williamson DH (1980). Physiological roles of ketone bodies as substrates and signals in mammalian tissues. Physiological Reviews 60: 143-187.

Roche Diagnostics (2013). Chemstrip 2 GP, 2 LN, 9, 10 with SG Urine Test Strips. Package insert No. 2013-11 V 3.0. Indianapolis, Roche Diagnostics.

Sleeman JM, Mudakikwa AB (1998). Analysis of urine from free-ranging mountain gorillas (Gorilla gorilla beringei) for normal physiologic values. Journal of Zoo and Wildlife Medicine 29: 432-434.

Vogel ER, Alavi SE, Utami-Atmoko SS, van Noordwijk MA, Bransford TD, Erb WM, Zulfa A, Sulistyo F, Farida WR, Rothman JM (2017a). Nutritional ecology of wild Bornean orangutans (Pongo pygmaeus wurmbii) in a peat swamp habitat: effects of age, sex, and season. American Journal of Primatology 79: 1-20.

Vogel ER, Bransford TD, Alavi SE, Emery Thompson M, Crowley BE, Erb WM, van Noordwijk MA, Utami-Atmoko SS, Raubenheimer D, Rothman JM (2017b). The power of protein: protein regulation, energetics, and health in wild Bornean orangutans (Pongo pygmaeus wurmbii). American Journal of Physical Anthropology 162 (S64): 397.

Vogel ER, Rothman JM, Moldawer AM, Bransford TD, Emery Thompson ME, van Noordwijk MA, Utami-Atmoko SS, Crowley BE, Knott CD, Erb WM, Raubenheimer D (2015). Coping with a challenging environment: nutritional balancing, health, and energetics in wild Bornean orangutans. American Journal of Physical Anthropology 156: 314-315.

Vogel ER, van Woerden JT, Lucas PW, Atmoko SSU, van Schaik CP, Dominy NJ (2008). Functional ecology and evolution of hominoid molar enamel thickness: Pan troglodytes schweinfurthii and Pongo pygmaeus wurmbii. Journal of Human Evolution 55: 60-74.

Williamson DH, Mellanby J, Krebs HA (1962). Enzymic determination of D- $\beta$-hydroxybutyric acid and acetoacetic acid in blood. Biochemical Journal 82: 90.

Williamson DH, Whitelaw E (1978). Physiological aspects of the regulation of ketogenesis. Biochemical Society Symposium 43: 137-161.

Validation of Ketone Urine Test Strips in Wild Bornean Orangutans
Folia Primatol 2020;91:159-167

DOI: $10.1159 / 000501933$ 